\title{
Automatic Generation, Creativity, and Production of Narrative Content
}

\author{
Takashi Ogata \\ Faculty of Software and Information Science, Iwate Prefectural University \\ 152-52, Sugo Takizawa, Iwate 020-0693, Japan \\ E-mail: t-ogata@iwate-pu.ac.jp
}

\begin{abstract}
In accordance with the concept of this organized session, in this presentation, we will consider the study of automatic narrative or story generation as a research field of artificial intelligence (AI) at the levels of generation, creation, and production. Firstly, we study the technologies of narrative or story generation systems based mainly on artificial intelligence and cognitive science. Next, the artistic and aesthetic problems of narrative creation are considered in terms of their relationships with technologies and systems of narrative generation. Further, issues of organizational or social narrative production are presented. The survey and discussion will be conducted based on topics in this session relating to game and advertising systems and our integrated narrative generation system.
\end{abstract}

Keywords: Multiple narrative structures model, Integrated narrative generation system (INGS), Geino information system (GIS), Narrative generation, Narrative production, Narrative creation.

\section{Introduction}

In this paper, I consider the study of automatic narrative or story generation as a highly interdisciplinary research field of information technologies, including artificial intelligence (AI) and cognitive science, as well as literary theories such as narratology.

For the author, this issue can be focused on the following levels: generation, production, and creation. The first automatic narrative "generation" means the technological aspect of systems that automatically generate narrative texts. In particular, it indicates a narrative generation mechanism as a single subject. In contrast, we call the narrative generation phenomenon at a collective and organizational level narrative "production." This differentiation is dependent upon a narrative model of multiple narrative structures as proposed by the author in the following section. The word "production" is closely associated with organizational or social narrative making. On the other hand, narrative "creation" is a word on a different level that has an artistic and aesthetic nuance.
This paper first presents the author's multiple narrative structures model as a background to this consideration. Next, we respectively explain the "Integrated Narrative Generation System: INGS"1 and "Geino Information System: GIS", as frameworks for single level narrative generation and multiple level narrative production. Lastly, according to the above frameworks, we discuss the possibilities of narrativebased systems and content technologies based on the relationships between narrative generation and production, future narrative creation at the artistic level, and aesthetic problems of narrative.

\section{A Model of Multiple Narrative Structures}

For the author, one of the basic concepts of narrative generation is "multiplicity." It is necessary to design narrative generation systems to model multiple narrative structures. Although such models have various aspects, from the formal aspect of narrative to the aspect of narrative semantics, an important characteristic is that narrative generation is divided into the following two 
aspects: (1) the narrative generation and reception process based on a single subject, and (2) the narrative production and consumption process based on many subjects or collective authors. In other words, the narrative production (and consumption) process contains many narrative generation (and reception) processes. The narrative production and consumption process is a field that is socially open and is strongly related to the documents and narratives produced by people on the web.

Further, in narratives, there exists narrative multiplicity among various elements, such as story and character. For instance, in the characters of kabuki plays, actors can appears with multiplicity, by, for example, playing one or several characters in the drama a contemporary person living in the modern day, a person from the past with an historical story, etc.

From the viewpoint of the author's "Expanded Literary Theory: ELT,"3 this model is partially related to Bakhtin's theory of polyphony ${ }^{4}$ and Kristeva's theory of inter-textuality. ${ }^{5}$ Bakhtin's theory terms as polyphonic novels those that are not integrated by a single authorial voice, while Kristeva's theory proposed that all types of document can respectively exist in a network with many documents and the fragments may include novels and other genres, whether or not the author is conscious of that fact. Both theories amplified narrative multiplicity in their respective manners. The multiple narrative structures model comprises these theoretical frameworks.

\section{Integrated Narrative Generation System (INGS)}

The INGS is a developing narrative generation architecture that corresponds to a single level narrative generation subject. Fig. 1 provides an overview of the INGS, demonstrating that various narrative techniques and knowledge are organized in a synthesizing system that associates a number of literary theories for the realization of ELT.

The INGS comprises several components related to the narrative generation process and control, narrative techniques for the generation of narrative structures, and narrative knowledge including relatively large-scale dictionaries.

At first, the generation process is divided into three phases: story, discourse, and expression. A "story" generated by the story generation mechanism means the content of a narrative or a temporal sequence of events. A story is represented by a tree structure consisting of the three basic units: event, relation, and state. A story structure is generated using one or more story techniques, and states associated with the events are created according to another mechanism. Through this processing, an event that is a basic element of a story and a discourse are also generated. Each event basically consists of a verb concept and some noun, and other concepts. A "discourse" transformed by the discourse mechanism signifies the structural aspect of narrating a story. The discourse structure and the surface media used to narrate it are distinguished and, accordingly, it is described with a conceptual representation form.

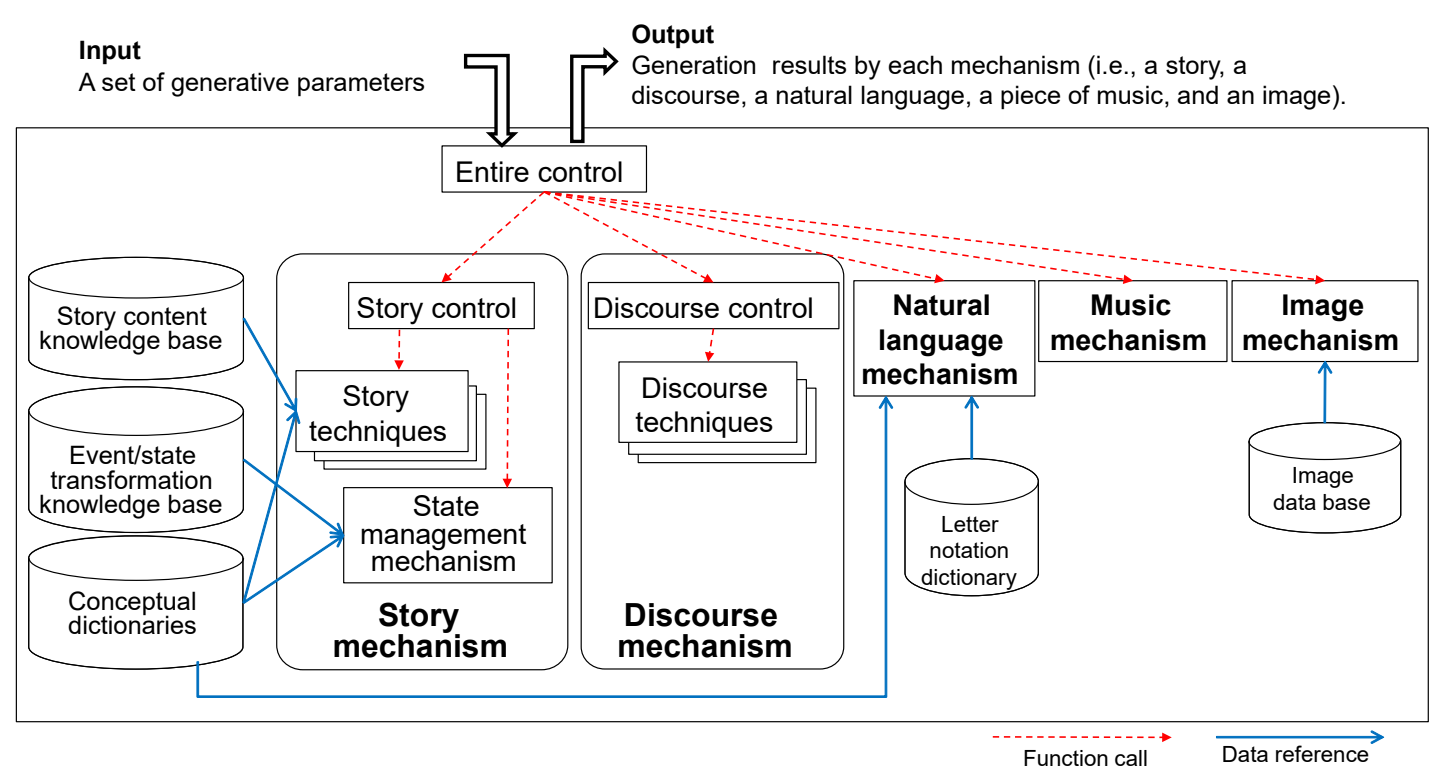

Fig. 1. An overall architecture of INGS ${ }^{1}$ 
"Expression" means the aspect of narrative representation by various surface media, including natural language, visual media, and music.

The control mechanism relating to many parts of the process currently utilizes a tentative approach based on the parameters and rules for the above three phases. In addition, a circulative process control, which does not limit the generation order among the phases to a fixed order, is also adopted in the meta-level mechanism.

The generation of each tree structure of a story and a discourse is performed using story techniques and discourse techniques that generate or transform from an old tree structure to a new tree structure using knowledge elements including conceptual dictionaries for verb and noun concepts. The surface narrative representation is conducted in this process.

An important design concept is "versatile," which means the potential to be used and applied to any purposes relevant to narrative generation without limitations regarding themes and genres. In the future perspective of the INGS, we aim to develop a versatile narrative generation architecture through which diverse narratives can be generated with various themes, genres, structures, etc. This would allow the INGS to be applied to various narrative production mechanisms as a single level narrative generation subject.

\section{Geino Information System}

The Japanese word geino has a complex meaning that includes elements of shrine rituals, entertainment, and amusement. From the viewpoint of narrative generation research, a geino system is a whole that multiply contains diverse narrative generation processes and comprises a multiple narrative phenomenon. For the author's research plan, the INGS is contained in the GIS

The GIS is a model in which multiple narrative generation and production mechanisms are conducted to perform the social level tasks of narrative. The INGS and GIS correspond to narrative generation-reception and narrative production-consumption respectively. In the GIS, various levels of the narrative generation process are driven by authors, receivers, characters, actors, and actresses. For example, a kabuki play is a collection of multiple narratives built around original scenarios, authentic related histories, and the actors' private scenes. The construction will be connected with the plan for a future narrative generation system comprised of several other narrative generation systems.

Fig. 2 shows an original conceptual version of the GIS $^{2}$. This idea of a conceptual model labelled GIS was originally based on a survey and analysis of Japanese folklore, performing arts including kabuki, and aspects of the modern entertainment business. A geino organization, such as an entertainment agency or promoter, is a synthetic narrative productionconsumption system that aggregates a variety of the narrative generation-reception processes of scenarios, the lives of actors and actresses, etc. The GIS is a comprehensive and multiple framework in which various narrative generation processes are driven by authors, receivers, characters, actors and actresses, directors, producers, etc. It is therefore one of the systems of multiple narrative structure.

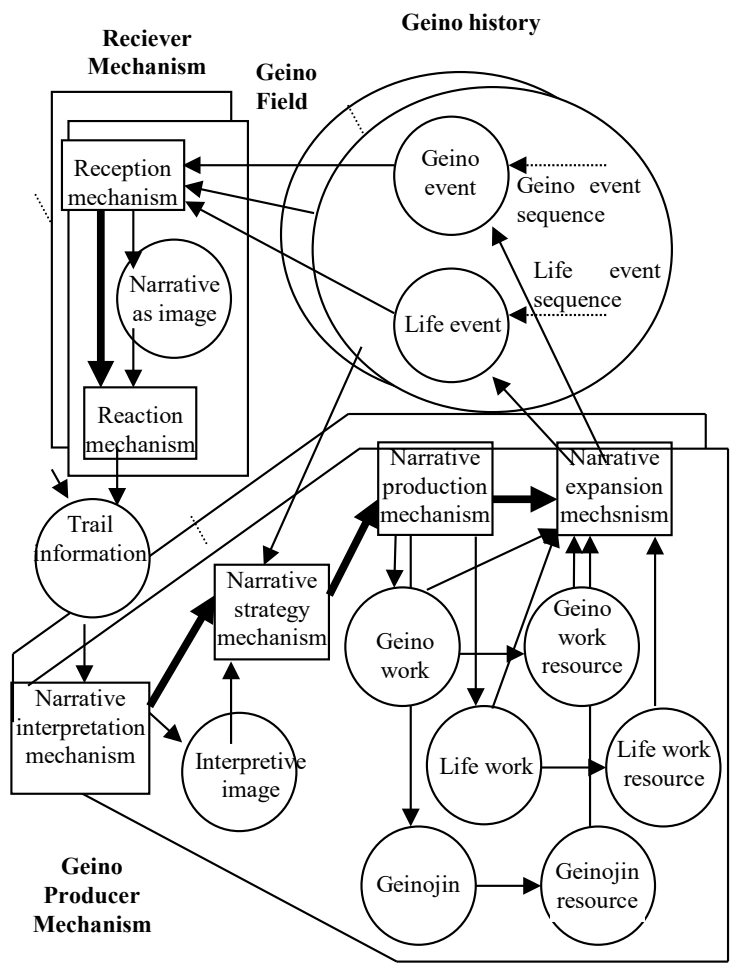

Fig. 2. The original GIS model ${ }^{2}$

\section{Generation, Production, and Creation of Narrative}

Although the INGS is a tentative but progressing system that is under development, the GIS remains at the conceptual level. The design and development of the GIS to bridge the narrative generation-reception and the production-consumption by the INGS is planned, based on the following process through development and experimentation with application systems. For instance, we have developed a multimedia narrative generation system called KOSERUBE, ${ }^{6}$ which was designed as an application using the INGS within the system. However, 
we do not intend to have a function through which the narrative generation and reception are repeated to store a kind of history of narrative generation. By designing such a circulative system, we will implement a prototype of the GIS based on KOSERUBE.

On the other hand, Kawamura ${ }^{7}$ and Ogata $^{8}$ have developed advertising generation systems based on original ideas. It is necessary for advertisement as a social system to have a history or story through its circulative generation, namely at the level of narrative production-consumption. In Ogata's study of advertisement as narrative, in particular, it will be possible to integrate the INGS or a part thereof as a single advertising generation mechanism into the GIS.

Such an approach to the GIS through narrative generation application systems will be possible in a variety of systems, such as entertainment game systems, ${ }^{9}$ a new narrative approach modelled on kabuki, and so on. Further, the narrative production and consumption model in which the GIS is included will link to the world of the contents business, including the results of narrative generation and the narrative generation function itself.

On the other hand, there is an important issue in another direction. By the word narrative "creation," we mean various values that go beyond the mere generation and production of narrative. In particular, the problem of narrative creativity is associated with diverse values, such as entertainment value, artistic value, literary value, aesthetic value, managerial value, etc. For instance, a contents business company related to narrative production needs to sell values by providing narrative content. For the author, this problem is related to the problem of narrative multiplicity or the multiple narrative structures model. We have an idea that narrative multiplicity results in narrative values. The verification of this idea or hypothesis will be attempted dependent upon the development and social practice of the GIS through various application systems.

\section{Conclusion}

We presented the author's multiple narrative structures model as a background to this consideration and introduced the INGS and GIS as frameworks for single level narrative generation and multiple level narrative production. Next, we discussed the possibilities of narrative-based systems and content technologies based on the relationships between narrative generation and production, further artistic level narrative creation and the aesthetic problems of narrative.

In the future, it is necessary to organically link the INGS and GIS as an integrated system; therefore, levels of narrative generation-reception and productionconsumption also need to be associated with each other. Further, the level of narrative creation should be considered in terms of the relationships of the above multiple system framework. Designing, developing, and experimenting (including at a social level) the INGS, GIS, and the application system are the main works.

\section{References}

1. T. Akimoto and T. Ogata, An Information Design of Narratology: The Use of Three Literary Theories in a Narrative Generation System, Int. J. Visual Design 7(3) (2014), pp.31-61.

2. T. Amino, Y. Kawamura and T. Ogata, Hierarchical Generation of Geino-Idol Stories: Toward Geino Information System and Narrative Marketing, Proc. $17^{\text {th }}$ Congress of the International Association of Empirical Aesthetics (2002), pp.549-552.

3. T. Ogata, Expanded Literary Theory for Automatic Narrative Generation, Proc. Joint 7th International Conference on Soft Computing and Intelligent Systems and 15th International Symposium on Advanced Intelligent Systems (2014), pp.1558-1563.

4. М. Bakhtin (М. М. Бахтин), Проблемы позтики Достоевского, Иэд, 2е. (Москва, 1963). Transl. С. Emerson, Problems of Dostoevsky's Poetics. (University of Minnesota Press, 1984).

5. J. Kristeva, Desire in Language: A Semiotic Approach to Literature and Art. (Columbia University Press, 1980).

6. S. Imabuchi, T. Akimoto, J. Ono and T. Ogata, KOSERUBE: An Application System with a Propp-based Story Grammar and Other Narrative Generation Techniques, Proc. the 6th International Conference on Soft Computing and Intelligent Systems \& the 13th International Symposium on Advanced Intelligent Systems (2012), pp.248-253.

7. Y. Kawamura, An Analysis of the Rhetoric of Commercial Film: Toward the Building of a Commercial Film Production Support System based on Image Rhetoric, Proc. the 2003 IEEE International Conference on Systems, Man and Cybernetics (Washington, DC, 2003), pp.993-1000.

8. Y. Zhang, J. Ono and T. Ogata, An Advertising Rhetorical Mechanism for Single Event Combined with Conceptual Dictionary in Narrative Generation System, 7th Int. Conf. on Natural Language Processing and Knowledge Engineering (2011), pp.340-343.

9. J. Ono and T. Ogata, Architecture of a Narrative Generation System based on a TRPG Model: The Use of an Integrated Narrative Generation System for Knowledge Acquisition (Preliminary Version), Bulletin of Networking, Computing, Systems, and Software 5(1) (2016) pp.40-48. 